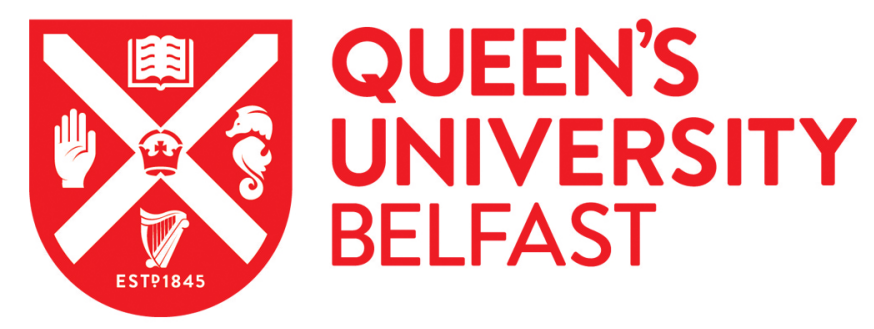

\title{
Electrostatic shock waves in the laboratory and astrophysics: similarities and differences
}

Dieckmann, M. E., Doria, D., Sarri, G., Romagnani, L., Ahmed, H., Folini, D., Walder, R., Bret, A., \& Borghesi, M. (2018). Electrostatic shock waves in the laboratory and astrophysics: similarities and differences. Plasma Physics and Controlled Fusion, 60(1), [014014]. https://doi.org/10.1088/1361-6587/aa8c8f

\section{Published in:}

Plasma Physics and Controlled Fusion

\section{Document Version:}

Peer reviewed version

Queen's University Belfast - Research Portal:

Link to publication record in Queen's University Belfast Research Portal

Publisher rights

( 2017 IOP Publishing Ltd. This work is made available online in accordance with the publisher's policies. Please refer to any applicable terms of use of the publisher.

\section{General rights}

Copyright for the publications made accessible via the Queen's University Belfast Research Portal is retained by the author(s) and / or other copyright owners and it is a condition of accessing these publications that users recognise and abide by the legal requirements associated with these rights.

Take down policy

The Research Portal is Queen's institutional repository that provides access to Queen's research output. Every effort has been made to ensure that content in the Research Portal does not infringe any person's rights, or applicable UK laws. If you discover content in the Research Portal that you believe breaches copyright or violates any law, please contact openaccess@qub.ac.uk. 


\title{
Electrostatic shock waves in the laboratory and astrophysics: similarities and differences
}

M.E. Dieckmann ${ }^{1}$, D. Doria ${ }^{2}$, G. Sarri ${ }^{2}$, L. Romagnani ${ }^{3}$, H. Ahmed $^{2}$, D. Folini ${ }^{4}$, R. Walder ${ }^{4}$, A. Bret ${ }^{5,6}$, and M. Borghesi ${ }^{2}$

1. Department of Science and Technology (ITN), Linköpings University, Campus Norrköping, SE-60174 Norrköping, Sweden

2. Centre for Plasma Physics (CPP), Queen's University Belfast, BT7 1NN, UK

3. École Polytechnique, CNRS, LULI, F-91128 Palaiseau, France

4. École Normale Supérieure, Lyon, CRAL, UMR CNRS 5574, Université de Lyon, France

5. ETSI Industriales, Universidad de Castilla-La Mancha, 13071 Ciudad Real, Spain

6. Instituto de Investigaciones Energéticas y Aplicaciones Industriales, Campus

Universitario de Ciudad Real, 13071 Ciudad Real, Spain

E-mail: mark.e.dieckmann@liu.se

\begin{abstract}
Contemporary lasers allow us to create shocks in the laboratory that propagate at a speed that matches that of energetic astrophysical shocks like those that ensheath supernova blast shells. The rapid growth time of the shocks and the spatio-temporal resolution, with which they can be sampled, allow us to identify the processes that are involved in their formation and evolution. Some laser-generated unmagnetized shocks are mediated by collective electrostatic forces and effects caused by binary collisions between particles can be neglected. Hydrodynamic models, which are valid for many large-scale astrophysical shocks, assume that collisions enforce a local thermodynamic equilibrium in the medium; laser-generated shocks are thus not always representative for astrophysical shocks. Laboratory studies of shocks can improve the understanding of their astrophysical counterparts if we can identify processes that affect electrostatic shocks and hydrodynamic shocks alike. An example is the nonlinear thin-shell instability (NTSI). We show that the NTSI destabilizes collisionless and collisional shocks by the same physical mechanism.
\end{abstract}


Electrostatic shock waves in the laboratory and astrophysics: similarities and differences 2

\section{Introduction}

The flow speed of the stellar wind, which is emanated by the hot corona of the sun, ranges from 250 to $750 \mathrm{~km} / \mathrm{s}$ [1]. Astrophysical winds or the blast shells emitted by supernovae can be even faster $[2,3]$. A wind or outflow expands until it encounters an obstacle or a second medium that moves at a different speed and can resist the wind's ram pressure. An example for an obstacle is a planetary magnetic field and a second medium could be the interstellar medium (ISM) [4]. Shocks form if the slowdown of the wind or outflow exceeds the speed of its density waves.

Let us consider the bow shock of the Earth that separates the magnetosheath from the solar wind [5]. The speed of the solar wind, measured in the rest frame of the Earth, exceeds the fast magnetosonic speed. The inflowing dilute upstream medium, which is the solar wind, is compressed and heated by the shock crossing and the required energy is extracted from its directed flow energy. The plasma of the shocked solar wind enters the magnetosheath, which is the bow shock's downstream region. The change of the density, temperature and speed of the medium across the shock is determined by the shock jump conditions, which are also known as the Rankine-Hugoniot equations [6].

The Rankine-Hugoniot equations were originally derived for shocks in a fluid or in a dense gas, which is an ensemble of microscopic charge-neutral particles that collide with their neighbours on spatio-temporal scales that are small compared to those of interest. These equations are based on the assumption that mass, momentum and energy are conserved across the shock and they are thus also valid for other materials. A magnetohydrodynamic (MHD) model and the therefrom obtained shock jump conditions can approximate plasma, in which Coulomb collisions between the particles occur frequently enough to keep the velocity distribution close to a Maxwellian one.

The low density and high temperature of the solar wind and of many astrophysical outflows imply that their material is fully ionized and binary collisions between the charged particles are negligble. The mean free path of a solar wind particle is, for example, longer than the distance between the Sun and the Earth. Many structures in the solar wind, such as coronal mass ejections [7] and the aforementioned bow shock, evolve on spatio-temporal scales that are small compared to the mean-free path and the inverse (Coulomb) collision frequency of particles in this medium. Fluid models do not always provide an accurate description for these structures. In practice MHD models are also valid on shorter scales because a thermal equilibrium in plasma is not only enforced by Coulomb collisions but also by plasma instabilities and by the interaction of charged particles with the plasma thermal noise [8, 9]. However, even these additional mechanisms can not establish a thermal equilibrium on the small spatial scales of the transition layers of the Earth's bow shock and of many astrophysical shocks.

If binary (Coulomb) collisions between plasma particles occur rarely and if the plasma dynamics is determined instead by the electromagnetic fields, which are driven by the ensemble of all charged particles, then we speak of a collisionless plasma. Shocks in such a plasma are called collisionless shocks and their dynamics is described by the 
kinetic equations. Even though shocks in collisionless and collisional fluids are sustained by different means, they can be affected by similar instabilities. The insight we gain by studying the instability in one medium can deepen our understanding of the instability in the other medium. This approach is useful if an instability is difficult to study either in a collisionless or in a collisional medium.

Laboratory astrophysics [10] aims at studying astrophysically relevant instabilities, structures and processes in the laboratory. It is not always clear how important collisions are in the laboratory plasma and in the astrophysical plasma. Instabilities that develop in collisionless and collisional plasma alike are thus interesting test cases for such experiments. We discuss here the nonlinear thin-shell instability (NTSI), which is an example for such an instability. The hydrodynamic NTSI [11, 12, 13] and its

MHD counterpart [14] affect pairs of shocks that enclose a thin fluid slab. They have been named as one of several instabilities that could break the radial symmetry of a supernova remnant (SNR) or destabilize the collision boundaries of colliding winds (See Refs. [11, 15, 16, 17, 18] for a discussion). The NTSI has never been systematically investigated in the laboratory for (magneto-)hydrodynamic flows.

Particle-in-cell (PIC) simulations [20, 21] and an experiment [19] suggest that the NTSI can also develop in collisionless plasma. Section 2 summarizes the PIC simulation technique, it discusses electrostatic shocks and the mechanism that is responsible for the collisionless NTSI. We present results in section 3 and discuss them in section 4.

\section{Background}

\subsection{PIC simulation code}

A PIC code approximates each plasma species $i$ by a phase space fluid $f_{i}(\mathbf{x}, \mathbf{v}, t)$ and represents it by an ensemble of computational particles (CPs) with the charge-to-mass ratio $q_{i} / m_{i}$, which must equal that of the approximated plasma species. Each CP $j$ has a position $\mathbf{x}_{j}$ and velocity $\mathbf{v}_{j}$. Moving charged particles induce electromagnetic fields. PIC codes compute these fields by adding up the partial currents of all CPs to the total current density $\mathbf{J}(\mathbf{x}, t)$, which updates the electromagnetic fields with Ampère's law

$$
\mu_{0} \epsilon_{0} \frac{\partial \mathbf{E}}{\partial t}=\nabla \times \mathbf{B}-\mu_{0} \mathbf{J}
$$

and with Faraday's law

$$
\frac{\partial \mathbf{B}}{\partial t}=-\nabla \times \mathbf{E}
$$

The field values are defined on a grid and both equations are approximated by suitable numerical schemes. The EPOCH code [22] we use fulfills Gauss' law and $\nabla \cdot \mathbf{B}=0$ to round-off precision. The field values are interpolated to the position $\mathbf{x}_{j}$ of each $\mathrm{CP}$ and its velocity is updated with the relativistic Lorentz force equation. The position of each CP is updated with its velocity and the time step. This computational cycle is repeated as often as necessary to cover the time scale of interest. 
Electrostatic shock waves in the laboratory and astrophysics: similarities and differences4

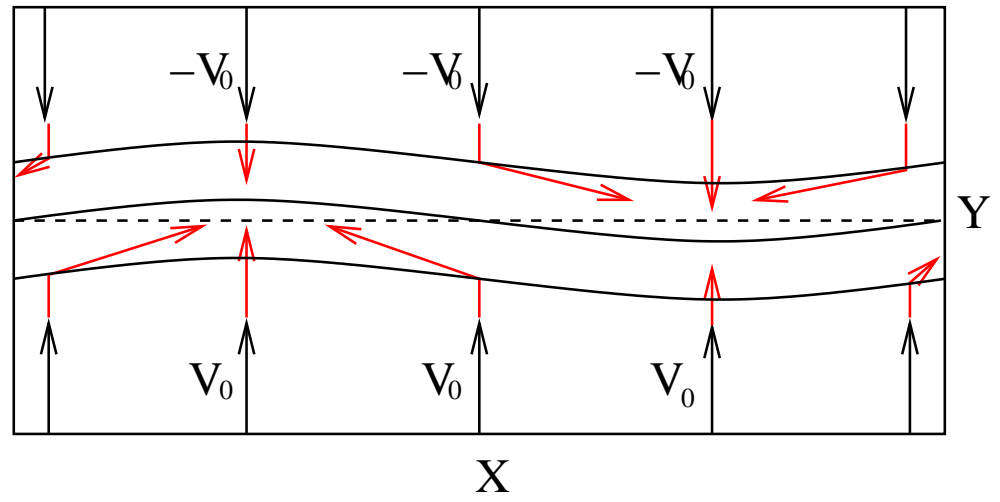

Figure 1. The NTSI: unmagnetized material streams in from below with the spatially uniform speed $\mathbf{v}_{0}$ and from above with the spatially uniform speed $-\mathbf{v}_{0}$. A thin shell is located in the center and its central curve is sinusoidally displaced relative to the horizontal dashed line. The two outer solid curves are hydrodynamic shocks in the case of a fluid and electrostatic shocks in the case of a collisionless plasma. The red broken arrows show how a fluid element or an ion is deflected when it crosses the shock. The rotation is accomplished by a reduction of the velocity normal to the shock by particle collisions or by the electric field that mediates the collisionless shock.

\subsection{The non-linear thin-shell instability (NTSI)}

Figure 1 illustrates the mechanism that drives the NTSI. Two hydrodynamic fluids or clouds of collisionless plasma flow towards a sinusoidal-shaped thin shell. Their velocity modulus $\left|\mathbf{v}_{0}\right|$ and density $n_{0}$ are spatially uniform on either side of the thin shell. The thin shell is composed of the shock-compressed downstream fluid and its outer boundaries are hydrodynamic shocks in the case of a collisional fluid. The shock crossing reduces the velocity component of the fluid along the shock normal.

If the inflowing material is collisionless plasma and if we neglect for the moment the interaction of the counterstreaming clouds through the collective electromagnetic fields then the thin shell is a layer with the density $2 n_{0}$, in which the ions of both clouds overlap. Each boundary between the thin shell and the inflowing plasma is characterized in this case by a density jump with the magnitude $n_{0}$. Thermal diffusion lets more electrons stream from the denser shell into the surrounding plasma than vice versa and the thin shell goes on a positive potential relative to the surrounding plasma. The ambipolar electric field, which sustains the potential difference, is aligned with the normals of the boundaries. This electric field slows down the inflowing ions in the rest frame of the thin shell, which compresses them and increases the plasma density in the thin shell beyond $2 n_{0}$. If the potential difference is comparable to the kinetic energy of the inflowing protons in the rest frame of the thin shell, then the boundaries are electrostatic shocks. An upstream proton (electron), which crosses the boundary and enters the thin shell, has its normal velocity reduced (increased). The opposite is true if the proton or electron leaves the thin shell and propagates back upstream. The latter is possible only in a collisionless plasma and the boundary acts in this case as a double 
layer. Hybrid structures that combine an electrostatic shock [25, 26, 27, 28, 29] with a double layer are common [30].

The lateral velocity components of the fluid elements or plasma particles remain unchanged and, hence, their velocity vectors are rotated. Figure 1 shows that fluid elements or protons that flow towards a concave boundary are focused while those flowing towards a convex boundary are scattered. In the case of a hydrodynamic thin shell Vishniac [11] has shown that this momentum redistribution applies a torque on a hydrodynamic thin shell, which amplifies the amplitude of the spatial oscillation. It also drives breather modes, which are density oscillations along the thin shell. In what follows we demonstrate that the same is true for a thin shell of collisionless plasma that is bounded by two electrostatic hybrid structures.

\subsection{Initial conditions}

We perform 4 simulations that resolve a two-dimensional domain with the length $L_{x}$ along $x$, which changes between simulations, and the fixed length $L_{y}$ along $y$. The initially unmagnetized plasma consists of electrons and protons with the spatially uniform number density $n_{0}$. We use the correct proton-to-electron mass ratio. We give the electrons the initial temperature $T_{0}=1500 \mathrm{eV}$ and the protons $T_{0} / 5$. Such a temperature difference is common if the plasma has been created by the ablation of a target by an energetic laser pulse [31] and these temperatures are comparable to those in the experiment in Ref. [19].

We normalize the spatio-temporal scales with the electron plasma frequency $\omega_{p}=$ $\left(e^{2} n_{0} / m_{e} \epsilon_{0}\right)^{1 / 2}\left(e, m_{e}, \epsilon_{0}\right.$ : elementary charge, electron mass and vacuum permittivity) and the electron Debye length $\lambda_{D}=v_{t e} / \omega_{p}$. The electron thermal speed is $v_{t e}=$ $\left(k_{B} T_{0} / m_{e}\right)^{1 / 2}$, where $k_{B}$ is the Boltzmann constant. The Debye length in the solar wind close to the Earth with the number density $\sim 10 \mathrm{~cm}^{-3}$ and temperature $5 \mathrm{eV}$ is about 5 meters and it is about $1 \mu \mathrm{m}$ in the experiment in [19]. Time is expressed as $t=\omega_{p} t_{p}$ and space as $x=x_{p} / \lambda_{D}$, where $t_{p}$ and $x_{p}$ are given in SI units. Electric and magnetic fields are normalized as $\mathbf{E}=e \mathbf{E}_{p} /\left(m_{e} v_{t e} \omega_{p}\right)$ and $\mathbf{B}=e \mathbf{B}_{p} /\left(m_{e} \omega_{p}\right)$. We normalize in all figures the proton speeds to the ion acoustic speed $c_{s}=\left(\left(\gamma_{e} k_{B} T_{0}+\gamma_{p} k_{B} T_{0} / 5\right) / m_{p}\right)^{1 / 2}\left(m_{p}\right.$ : proton mass). We assume that the electrons have three degrees of freedom $\left(\gamma_{e}=5 / 3\right)$ and the protons one $\left(\gamma_{p}=3\right)$ giving $c_{s} \approx 5.7 \times 10^{5} \mathrm{~m} / \mathrm{s}$ or $c_{s} / v_{t e} \approx 0.035$.

We resolve the electrons and protons by 100 CPs per cell respectively and the interval $-L_{y} / 2 \leq y \leq L_{y} / 2$ with $L_{y}=660$ by 1500 grid cells. We employ open boundaries along $\mathrm{y}$ and periodic ones along $\mathrm{x}$. Two plasma clouds are placed in the simulation box, which are separated at the time $t=0$ by the boundary $y_{B, i}(x)=$ $A_{i} \sin \left(2 \pi x / L_{x}\right)$. The electrons and protons of the cloud with $y<y_{B, i}(x)$ have the mean speed $v_{0}=1.93 c_{s}$ along $\mathrm{y}$ and those in the cloud with $y>y_{B, i}(x)$ have the speed $-v_{0}$ along $\mathrm{y}$. In what follows we examine only the proton distributions because $v_{0} / v_{t e}=0.068$ implies that the electrons do not participate in the plasma dynamics apart from providing the thermal pressure that lets the shocks form. Both plasma 
Electrostatic shock waves in the laboratory and astrophysics: similarities and differences6

\begin{tabular}{|l|l|l|l|l|} 
Label & Simulation 1 & Simulation 2 & Simulation 3 & Simulation 4 \\
\hline$L_{x}$ in $\lambda_{D}$ & 110 & 110 & 110 & 55 \\
Grid cells & 250 & 250 & 250 & 125 \\
$A_{i}$ in $\lambda_{D}$ & 0 & 0.9 & 2.2 & 2.2 \\
\hline
\end{tabular}

Table 1. The initial conditions that varied between the simulations. The seed perturbation for the NTSI is given in all simulations by the initial collision boundary $y_{B, i}(x)=A_{i} \sin \left(2 \pi x / L_{x}\right)$.

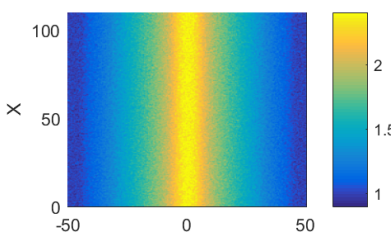

(a) $Y$

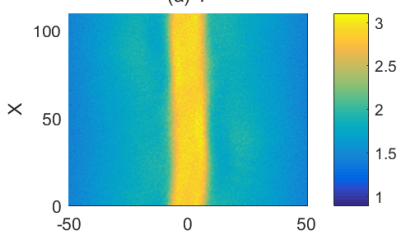

(e) $Y$

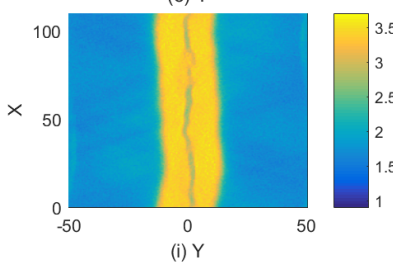

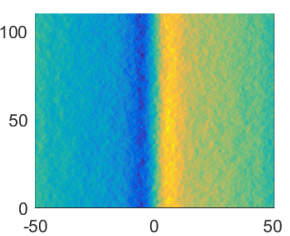

(b) $Y$

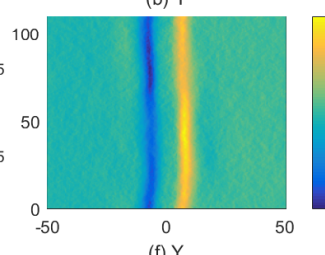

f) $Y$

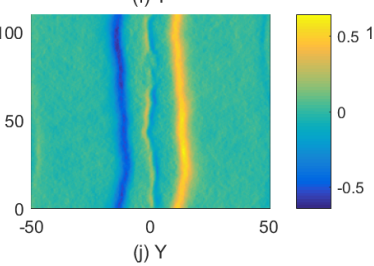

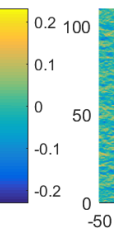

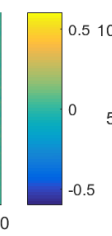

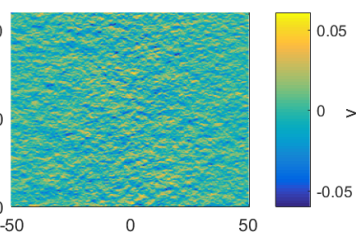

(c) $Y$

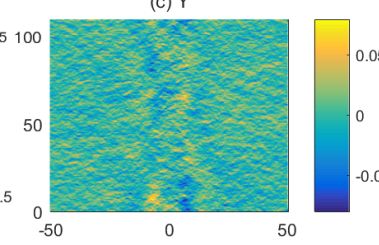

(g) $Y$

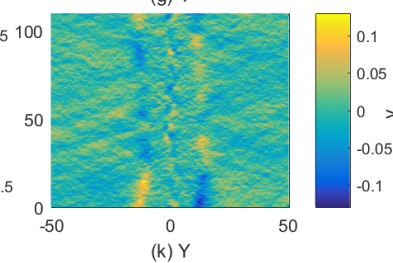

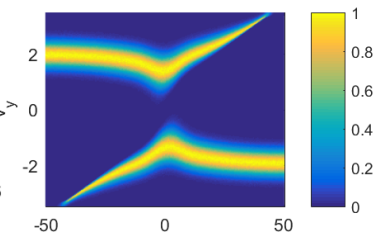

(d) $Y$

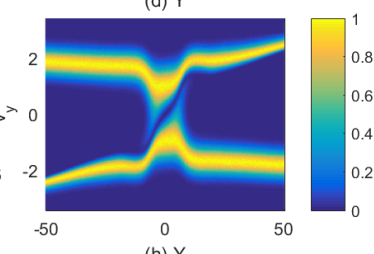

(h) $Y$

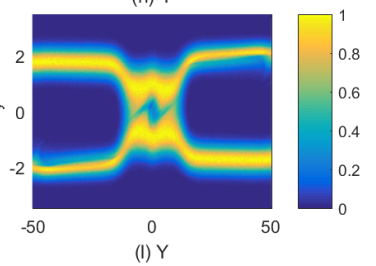

Figure 2. The plasma evolution in simulation 1: the first row corresponds to the time $t=400$, the second row to $t=800$ and the bottom row to $t=1600$. The columns from the left to the right show the proton density, the electric $E_{y}$ component, the electric $E_{x}$ component and the x-averaged proton phase space density distribution $f_{p}\left(y, v_{y}\right)$.

clouds interpenetrate at $y_{B, i}$ and in time a plasma density distribution develops, which resembles that in Fig. 1. All simulations evolve the collision during the time $t_{s}=1600$, which is resolved by $10^{5}$ time steps. Table 1 lists the other parameters.

\section{The simulations}

\subsection{Planar electrostatic shock}

Figure 2 shows the distributions of the electromagnetic fields, of the proton density and of the proton phase space density at selected times, which have been computed by a simulation with $A_{1}=0$ and, hence, $y_{B, 1}=0$.

Time $t=400$ : protons have accumulated close to $y=0$ in Fig. 2(a) and the interval with an increased proton density extends to $|y| \approx 45$. The peak density 2.3 close to $y=0$ implies that the protons have been compressed beyond the density, which we would expect from the superposition of the densities of both plasma clouds. We will 
refer to the central high-density band as the thin shell and to the surrounding region with a density $>1$ as the overlap layer. Figure 2(b,c) show the in-plane electric field components $E_{y}$ and $E_{x}$, which are driven by the thermal diffusion of electrons against a density gradient. This ambipolar electric field is aligned with $y$ since $E_{x}=0$.

We observe already at this time a significant slowdown of the inflowing protons in Fig. 2(d). The velocity oscillation in each proton beam close to $y=0$ is a solitary wave, which will continue to grow into an electrostatic shock [23, 24]. The kinetic energy of all protons exceeds the potential energy they gain as they approach the center of the thin shell and the counterstreaming proton beams remain compact. The protons cross the thin shell and are re-accelerated by the ambipolar electric field while they leave it on the other side. They are further accelerated by the weaker electric field that arises from the density gradient of the overlap layer, which extends far beyond the thin shell. The selected collision speed exceeds the maximum speed, for which the potential jump that is associated with the density jump $\sim 1$ can sustain a pair of shocks. However, the slow-down of the protons compresses them to a density above 2 in the thin shell, which increases the potential gap between the dense thin shell and the surrounding plasma and leads to a further compression of the protons. This feedback loop results in a delayed shock formation as long as the collision speed is not too high [29]. Otherwise the shock formation requires the growth of an instability that increases the thermal and magnetic pressure or generates magnetic fields that can block the plasma flow [32].

Time $t=800$ : the feedback loop has increased the peak proton density to over 3 as we can see from Fig. 2(e). Two unipolar narrow electric field pulses delimit the thin shell and the electric field is aligned with the density gradient (See Figs. 2(f,g)). Figure 2(h) shows that the proton speed changes rapidly at the location of the electric field pulse. The velocity spread of the inflowing proton beams at $|y| \approx 5$, which is a consequence of their slowdown by the electric field, is so large that some protons change the sign of their velocity. These protons will feed the shock-reflected proton beam, which is a signature of an electrostatic shock in warm protons.

Time $t=1600$ : the proton density in Fig. 2(i) has reached a peak value of 3.6 and it has expanded along $y$, which is only possible if the thin shell is accumulating protons. The electric field in Figs. $2(\mathrm{j}, \mathrm{k})$ is still aligned with the density gradient but both are no longer planar. The bipolar electric field distribution close to $y \approx 0$ is a forming proton phase space hole [33]. A dilute population of protons is gyrating in the $\left(y, v_{y}\right)$ plane close to $y=0$ and $v_{y}=0$ in Fig. 2(l). The proton beams have a constant velocity outside $|y|=20$ in the displayed interval and they change their speed only at the electrostatic shock when they enter the thin shell and at the double layer, where they exit the thin shell and escape into the overlap layer. The thin shell consists of a hot proton population and two dense counterstreaming proton beams, which are separated by a small velocity gap. An ion-beam driven instability [34] will eventually thermalize the proton beams inside the thin shell [35] and in the overlap layer [27, 28]. 
Electrostatic shock waves in the laboratory and astrophysics: similarities and differences8
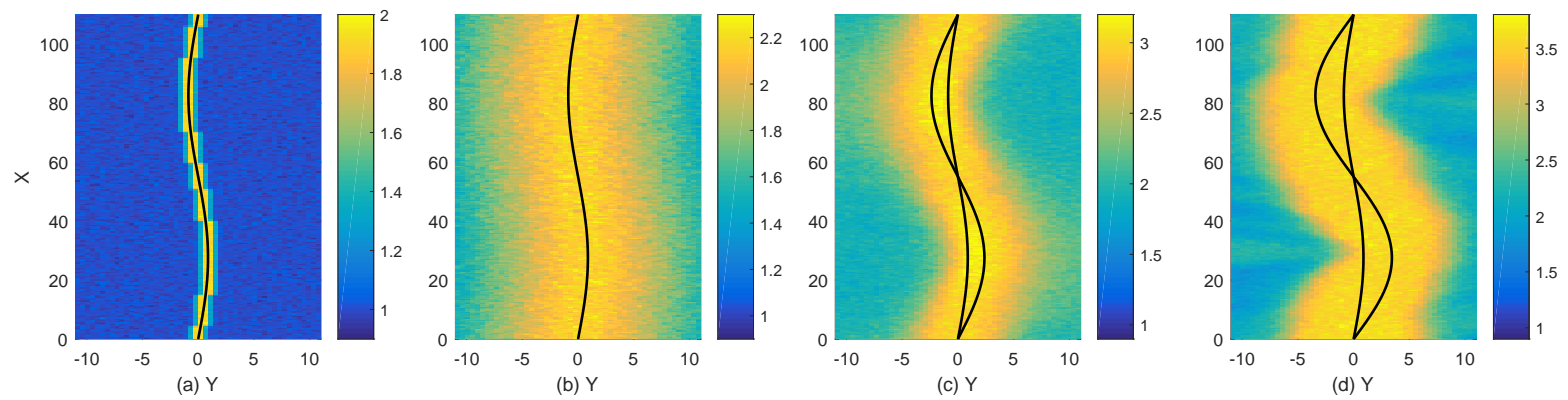

Figure 3. The proton density distribution at the times $t_{1}=8$ (a), $t_{2}=177$ (b), $t_{3}=813(\mathrm{c})$ and $t_{4}=1600(\mathrm{~d})$ computed by simulation 2 . The seed oscillation $y_{B, 2}(x)$ is overplotted in all panels. The curve $y_{2}\left(x, t_{3}\right)=2.7 \cdot y_{B, 2}(x)$ is overplotted in (c) and $y_{2}\left(x, t_{4}\right)=3.9 \cdot y_{B, 2}(x)$ is overplotted in $(\mathrm{d})$.

\subsection{Varying the perturbation amplitude $A_{i}$}

We compare first the results of simulations 2 and 3 that use the same value $L_{x}=110$ and, hence, the same wavelength $k_{i}=2 \pi / L_{x}$ of the seed perturbation for the NTSI. Vishniac estimated in Ref. [11] the growth rate of the NTSI as $\omega_{v, i} \sim c_{s} k_{i}\left(k_{i} A_{i}\right)^{1 / 2}$ ( $i$ : simulation number), provided that the seed amplitude $A_{i}$ is above the threshold for instability and that $A_{i} \ll L_{x}$. We obtain for $A_{2}$ the value $t_{s} \omega_{v, 2} / \omega_{p} \approx 0.77\left(t_{s}=1600\right)$ while $A_{3}$ gives $t_{s} \omega_{v, 3} / \omega_{p} \approx 1.14$. We expect to see at least the onset of the NTSI in both simulations if Vishniac's growth rate estimate holds also for collisionless plasma.

Several proton density distributions, which were computed by simulation 2, are

shown in Fig. 3. The function $y_{2}(x, t)=H\left(t-t_{2}\right) \sqrt{\left(0.08 \cdot\left(t-t_{2}\right)+1\right)} y_{B, 2}(x)$ with $y_{B, 2}(x)=A_{2} \sin \left(2 \pi x / L_{x}\right)$ is a fit to the central curve of the thin shells, where $H(t)$ is the Heaviside step function and $t_{2}=177$ is the time when the NTSI sets in. The term $\propto \sqrt{m\left(t-t_{2}\right)+1}(m$ : slowly varying factor) from Ref. [36] matches that observed in the PIC simulation in Ref. [19] and in the present simulations. A sinusoidal-shaped thin shell has formed in Fig. 3(a) around $y_{B, 2}(x)$. It has expanded to a width $\sim 10$ in Fig. 3(b). Its boundaries still follow $y_{B, 2}(x)$ and the NTSI has not yet developed. The growth of a solitary wave in each proton beam lets the peak density of the thin shell grow beyond 2 after $t=t_{2}$. The supplementary movie 1 shows that the NTSI starts to grow at this time and the oscillations of the thin shell's boundary have reached $2.7 A_{2}$ at the time $t=813$ in Fig. 3(c). A sine curve with the amplitude $3.9 A_{2}$ approximates well the central curve of the thin shell in Fig. 3(d). The boundaries of the thin shell are no longer sine curves at $t=1600$. An ion acoustic instability develops upstream of the electrostatic shocks, which starts to disrupt the thin shell's boundaries [21].

Figure 4 shows the proton density distribution computed by simulation 3 for the same times that were shown in Fig. 3. The curve $y_{3}(x, t)=A_{3} y_{2}(x, t) / A_{2}$ is overplotted for all times. The proton density distribution is animated in time by the supplementary movie 2. The NTSI sets in at about the same time in the simulations 2 and 3, which suggests that it grows once the solitary waves have reached a threshold amplitude. The growth rate of the NTSI is about the same in both simulations, which is not what we 
Electrostatic shock waves in the laboratory and astrophysics: similarities and differences9
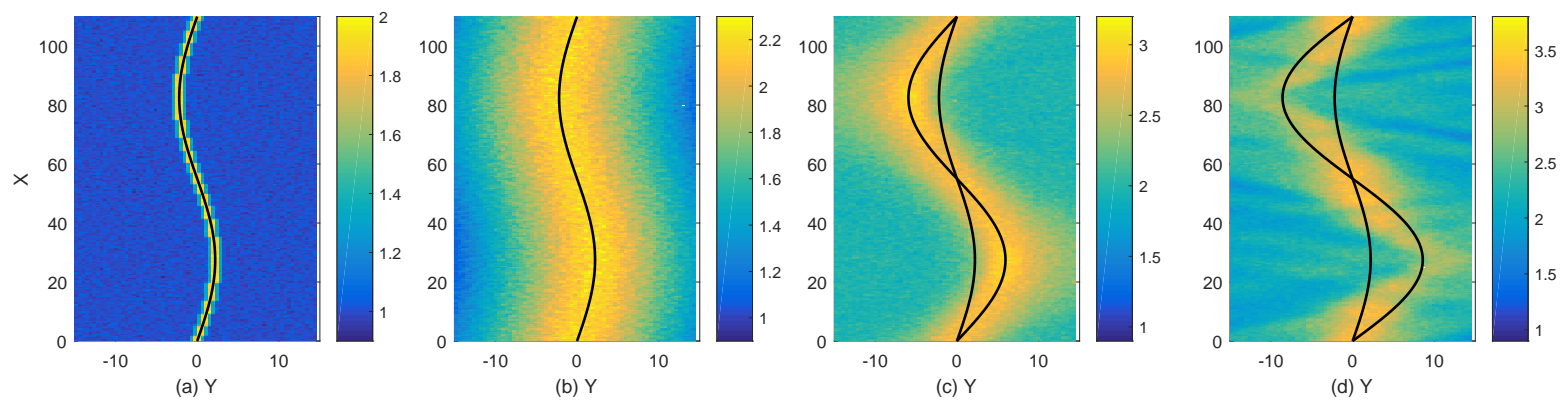

Figure 4. The proton density distribution at the times $t_{1}=8$ (a), $t_{2}=177$ (b), $t_{3}=813(\mathrm{c})$ and $t_{4}=1600(\mathrm{~d})$ computed by simulation 3 . The seed oscillation $y_{B, 3}(x)$ is overplotted in all panels. The curve $y_{3}\left(x, t_{3}\right)=2.7 \cdot y_{B, 3}(x)$ is overplotted in (c) and $y_{3}\left(x, t_{4}\right)=3.9 \cdot y_{B, 3}(x)$ is overplotted in (d).

expect from Vishniac's growth rate estimate $\propto A_{i}^{1 / 2}$. However, our fit of $y_{2}(x, t)$ and $y_{3}(x, t)$ to the simulation data may not be accurate enough to resolve a growth rate difference $\left(A_{3} / A_{2}\right)^{1 / 2} \approx 1.5$.

Vishniac found in Ref. [11] that the hydrodynamic NTSI drives breathing modes that yield density oscillations along the thin shell. The collisionless analogue of these hydrodynamic density waves are ion acoustic oscillations, which have the phase speed $\sim c_{s}$. Breather modes can explain why the thin shell in Fig. 4(d) has been depleted close to the extrema $x \approx 27$ and $x \approx 80$ of its oscillation while protons accumulated at these locations in Fig. 4(c). Let us assume that an ion acoustic oscillation develops between two extrema of the thin shell's oscillation, which are separated by a distance 55 along the thin shell. A full oscillation period in time is about $\Delta \approx 55 v_{t e} / c_{s} \approx 1600$. Initially the proton density is constant along the thin shell. The protons start to be deflected towards the extrema of the thin shell when the NTSI sets in at $t \approx t_{2}$ and the breather mode starts to grow. The proton density peaks at the extrema of the thin shell after one fourth of the breather mode's oscillation and, thus, at $t \approx t_{2}+\Delta_{t} / 4$, which is confirmed by the supplementary movie 2 . The breather mode will result in proton density minima at the extrema of the thin shell after $t_{2}+3 \Delta_{t} / 4 \approx 1400$, which explains the density distribution observed in Fig. 4. The breather mode was found also for a different set of initial conditions for the PIC simulations [21].

\subsection{Varying the perturbation wavelength $L_{x}$}

The hydrodynamic growth rate scales as $L_{x}^{-3 / 2}$ with the wavelength of the seed perturbation and we determine with simulation 4 how the growth of the bending mode and the nonlinear evolution of the collisionless NTSI are affected by halving $L_{x}$. The supplementary movie 3 demonstrates that the NTSI lets the central curve of the thin shell evolve as $y_{4}(x, t)=H\left(t-t_{2}\right) \sqrt{\left(0.56 \cdot\left(t-t_{2}\right)+1\right)} y_{B, 4}(x)$.

Figure 5 shows four snapshots of the proton density distribution. The thin shell in Fig. 5(a) follows the overplotted curve $y_{B, 4}(x)$ and it has expanded again to a width of about 10 at $t=t_{2}$. The NTSI sets in after this time and the amplitude of the oscillation 
Electrostatic shock waves in the laboratory and astrophysics: similarities and differences10
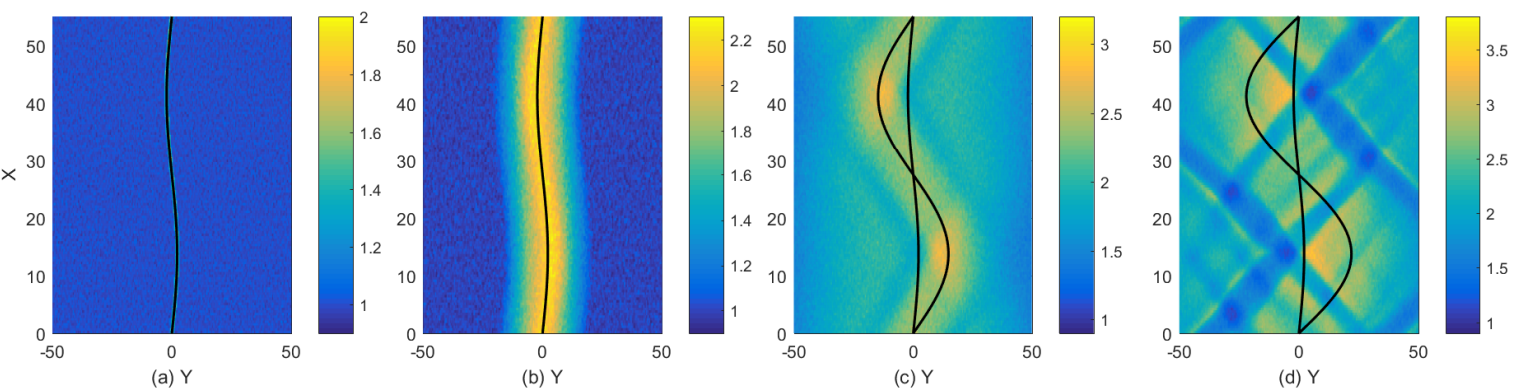

Figure 5. The proton density distribution at the times $t_{1}=8$ (a), $t_{2}=177$ (b), $t_{3}=813(\mathrm{c})$ and $t_{4}=1600(\mathrm{~d})$ computed by simulation 4 . The seed oscillation $y_{B, 4}(x)$ is overplotted in all panels. The curve $y_{4}\left(x, t_{3}\right)=6.7 \cdot y_{B, 4}(x)$ is overplotted in (c) and $y_{4}\left(x, t_{4}\right)=10 \cdot y_{B, 4}(x)$ is overplotted in $(\mathrm{d})$.
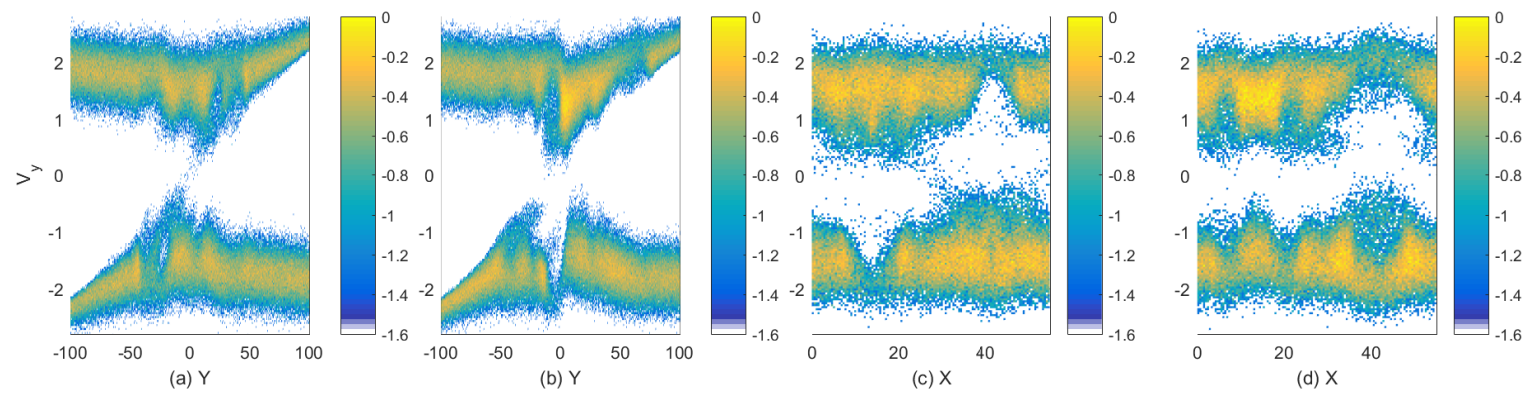

Figure 6. The proton phase space density distribution at the times $t_{4}=1600$ computed by simulation 4. Panel (a) shows the distribution along the slice $x=0$ and (b) shows it along $x=13.75$. Panel (c) shows the distribution along $y=0$ and (d) that along $y=10$. The color scale is 10-logarithmic and panels (a,b) and panels $(\mathrm{c}, \mathrm{d})$ are normalized to the peak value in the pair of panels.

has grown to $3.9 A_{4}$ by $t=t_{3}$. We observe a strong proton accumulation at the extrema of the thin shell at $x=13$ and $x=44$ and the density decreases from a peak value of 3.3 to a value of 2.3 over just a few electron Debye lengths. The thermal pressure gradient associated with these density changes will lead to changes in the potential that are comparable to that between the thin shell and the plasma far upstream. The protons respond to this ambipolar electric fields and a complex proton density distribution has developed at $t=t_{4}$, which is quasi-stationary (See supplementary movie 3 ).

We can shed light on the cause of these density structures with the help of Fig. 6, which shows slices of the proton phase space density distribution along $x$ and along $y$. Figure $6(\mathrm{a}, \mathrm{b})$ show the proton phase space density distributions along $y$ with $x=0$, for which $y_{B, 4}(x)=0$, and along $y$ with $x=13.75$, for which $y_{B, 4}(x)$ attained its largest value 2.2. The distributions of the counterstreaming clouds are symmetric in Fig. 6(a) and they are not symmetric in Fig. 6(b). This symmetry break can be linked to the motion of the thin shell in the simulation frame. The thin shell at $x=13.75$ propagates from $y \approx 2.2$ at $t_{2}$ to $y \approx 15$ at $t_{2}$, which corresponds to an average speed of $0.56 c_{s}$. The motion of the thin shell's electric potential to increasing $y$ implies that it affects 
the protons, which move in the opposite direction, less than the comoving ones. The counter-streaming proton clouds are well separated along $v_{y}$, while we could observe at this time a fully developed shock in simulation 1 (See Fig. 2(l)). The modulation of the solitary wave by the NTSI apparently inhibits or delays the shock formation.

The slice of the proton phase space density distribution along $x=0$ in Fig. 6(c) shows a depletion of the beam with $v_{y} \approx 1.9 c_{s}$ at $x \approx 43$ and of the oppositely propagating proton beam at $x \approx 13.75$. The accumulation of protons at these values of $x$ and $y \approx 0$ in Fig. $5(\mathrm{~d})$ drives ambipolar electric fields, which are tilted relative to the $y$ axis. The protons that move to increasing $y$ are scattered by the oblique electric fields at $x=43$ and $y<0$, which explains their depletion at $y=0$ in Fig. 6(c). The scattering of the protons will broaden this depletion with increasing $y>0$, which is confirmed by Fig. 6(d). The proton density distribution in Fig. 6(d) is caused by localized tilted electric fields and the ballistic motion of protons in the field-free intervals. The supplementary movie 3 shows that the distribution reaches a stable final state.

\section{Summary}

Here we have discussed some aspects of the non-linear thin-shell instability (NTSI). This instability was found in Ref. [11] for thin shells, which are enclosed by a pair of corrugated hydrodynamic shocks in a cool isothermal flow. It is believed to be important for the generation of turbulence in colliding winds and in regions where astrophysical outflows, such as the blast shells of supernova explosions, interact with the interstellar medium. This instability was examined with numerical simulations using MHD and hydrodynamic codes $[14,15]$ and it was observed in a recent laser-plasma experiment [19], where it deformed a thin shell of collisionless plasma. The PIC simulations in Refs. $[19,20,21]$ have shown that a collisionless analogue of the NTSI exists. A thin-shell, which is bounded by two solitary waves that are growing into electrostatic shocks [24], is destabilized by a spatial displacement of its central curve along the plasma flow direction that varies as a function of the orthogonal direction.

We have illustrated with a PIC simulation how a thin shell of dense plasma forms close to a planar collision boundary between two plasma clouds, which collide head on and orthogonal to the boundary, and how a pair of electrostatic shocks grows that separates the inflowing upstream protons from those in the thin shell. The absence of binary collisions between particles allowed some of the protons to traverse the thin shell and escape from it at the other side, while being re-accelerated by a double layer.

We have kept the collision speed the same and we performed 3 simulations, in which we seeded the collision boundary with a sinusoidal perturbation. Varying the amplitude and wave length of this perturbation allowed us to test how the growth rate of the collisionless NTSI and its non-linear evolution depended on the seed perturbation. The growth rate hardly changed with the amplitude of the seed perturbation, while a reduction of its wavelength clearly accelerated its growth. The amplitude grew in all cases with approximately $\sqrt{m\left(t-t_{2}\right)+1}$ with a constant $m$. 
The collisionless NTSI drove bending modes and breathing modes, which is also the case for the hydrodynamic one. The breathing modes involved large electric fields if the wavelength of the seed perturbation was short and we recovered here the complex spatial plasma density distribution from Ref. [20]. These structures could not develop in the case of the large initial seed wavelength due to the weaker electric field and a slower growth and we obtained the final proton density distribution from Refs. [20, 19].

We acknowledge support by a visiting fellowship of CRAL, by the EPSRC grants: $\mathrm{EP} / \mathrm{P} 010059 / 1, \mathrm{EP} / \mathrm{K} 022415 / 1$ and EP/N027175/1 and by the grant ENE2016-75703-R from the Spanish Ministerio de Educación. The simulations were performed on resources provided by the Grand Equipement National de Calcul Intensif (GENCI) through grant x2014046960. Some simulations were performed on resources provided by the Swedish National Infrastructure for Computing (SNIC) at the HPC2N computer centre.

\section{References}

[1] Hansteen V H and Velli M 2012 Space Sci. Rev. 17289

[2] Vink J 2011 Astron. Astrophys. Rev. 2049

[3] Marcowith et al. 2016 Rep. Prog. Phys. 792016

[4] K M Ferriere 2001 Rev. Mod. Phys. 73103

[5] Burgess D, Mobius E and Scholer M 2012 Space Sci. Rev. 1735

[6] Petrinec S M and Russel C T 1997 Space Sci. Rev. 79757

[7] Low B C 1996 Solar Phys. 167217

[8] Dupree T H 1963 Phys. Fluids 61714

[9] Bret A 2015 J. Plasma Phys. 81455810202

[10] Remington B A, Drake R P and Ryutov D D 2006 Rev. Mod. Phys. 78755

[11] Vishniac E T 1994 Astrophys. J. 428186

[12] Blondin J M and Marks B S 1997 New Astron. 1235

[13] Lamberts A and Fromang S and Dubus G 2011 Mon. Not. R. Astron. Soc. 4182618

[14] McLeod A D and Whitworth A P 2013 Mon. Not. R. Astron. Soc. 431710

[15] Blondin J M, Wright E B, Borkowski K J and Reynolds S P 1998 Astrophys. J. 500342

[16] Folini D and Walder R 2006 Astron. Astrophys. 4591

[17] van Marle A J, Keppens R and Meliani Z 2011 Astron. Astrophys. 527 A3

[18] van Marle A J and Keppens R 2012 Astron. Astrophys. 547 A3

[19] Ahmed H et al. 2017 Astrophys. J. Lett. 834 L21

[20] Dieckmann M E, Ahmed H, Doria D, Sarri G, Walder R, Folini D, Bret A, Ynnerman A and Borghesi M 2015 Phys. Rev. E 92031101

[21] Dieckmann M E, Folini D and Walder R 2017 Mon. Not. R. Astron. Soc. 4654240

[22] Arber T D et al. 2015 Plasma Phys. Controll. Fusion 57113001

[23] Ahmed H et al. 2013 Phys. Rev. Lett. 110205001

[24] Liseykina T V, Dudnikova G I, Vshivkov V A and Malkov M A 2015 J. Plasma Phys. 81495810507

[25] Forslund D W and Shonk C R 1970 Phys. Rev. Lett. 251699

[26] Forslund D W and Freidberg J P 1971 Phys. Rev. Lett. 271189

[27] Karimabadi H, Omidi N and Quest K B 1991 Geophys. Res. Lett. 181813

[28] Kato T N and Takabe H 2010 Phys. Plasmas 17032114

[29] Dieckmann M E, Ahmed H, Sarri G, Doria D, Kourakis I, Romagnani L, Pohl M and Borghesi M 2013 Phys. Plasmas 20042111

[30] Hershkowitz N 1981 J. Geophys. Res. 863307

[31] Eidmann K, Meyer-ter-Vehn J and Schlegel T 2000 Phys. Rev. E 621202 
[32] Novo A S, Bret A, Fonseca R A and Silva L O 2015 Astrophys. J. Lett. 803 L29

[33] Eliasson B and Shukla P K 2006 Phys. Rep. 422225

[34] Forslund D W and Shonk C R 1970 Phys. Rev. Lett. 25281

[35] Dieckmann M E, Sarri G, Doria D, Pohl M and Borghesi M 2013 Phys. Plasmas 20102112

[36] Doria D, Bret A and Dieckmann M E 2017 arXiv:1611.04454 